\title{
Active-Current Control of Large-Scale Wind Turbines for Power System Transient Stability Improvement Based on Perturbation Estimation Approach
}

\author{
Peng Shen *, Lin Guan, Zhenlin Huang, Liang Wu and Zetao Jiang \\ School of Electrical Power, South China University of Technology, Guangzhou 510641, China; \\ lguan@scut.edu.cn (L.G.); h.zhenlin@mail.scut.edu.cn (Z.H.); scutwuliang@foxmail.com (L.W.); \\ leo19930731@hotmail.com (Z.J.) \\ * Correspondence: shen.p@mail.scut.edu.cn; Tel.: +86-135-6012-1073
}

Received: 1 July 2018; Accepted: 30 July 2018; Published: 1 August 2018

\begin{abstract}
This paper proposes an active-current control strategy for large-scale wind turbines (WTs) to improve the transient stability of power systems based on a perturbation estimation (PE) approach. The main idea of this control strategy is to mitigate the generator imbalance of mechanical and electrical powers by controlling the active-current of WTs. The effective mutual couplings of synchronous generators and WTs are identified using a Kron-reduction technique first. Then, the control object of each WT is assigned based on the identified mutual couplings. Finally, an individual controller is developed for each WT using a PE approach. In the control algorithm, a perturbation state (PS) is introduced for each WT to represent the comprehensive effect of the nonlinearities and parameter variations of the power system, and then it is estimated by a designed perturbation observer. The estimated PS is employed to compensate the actual perturbation, and to finally achieve the adaptive control design without requiring an accurate system model. The effectiveness of the proposed control approach on improving the system transient stability is validated in the modified IEEE 39-bus system.
\end{abstract}

Keywords: wind turbine; power system transient stability; perturbation estimation; effective mutual coupling; perturbation observer

\section{Introduction}

Transient stability, which reflects the ability of power systems in maintaining synchronism when subjected to a severe fault, is an important factor in the design and operation of power systems [1]. A transient stability control strategy (TSCS) is essential to prevent a system from losing synchronism. The traditional TSCS includes generator tripping [2], load shedding [3,4], and excitation boosting control [5], etc. However, the improvement provided by the excitation boosting control is limited due to the restriction of maximum exciting current. The generator tripping and load shedding would bring about undesired economic loss. Considerable efforts are still devoted to finding effective and economical TSCSs to improve the system transient stability.

The penetration level of wind power in systems has increased significantly around the world during the past decades [6,7]. Among the new installed wind turbines (WTs), the doubly fed induction generator (DFIG) is the most popular technology for high power conversion efficiency and low manufacturing cost $[8,9]$. The DFIGs are connected to the system though power electronic converters and could provide flexible controllability. Although the integration of large-scale WTs could either benefit or deteriorate the system transient stability, depending on the actual situation, transient stability can be improved significantly by applying a supplementary control strategy on WTs $[10,11]$. 
Reference [11] proposed a supplementary control strategy to modulate the active-power of WTs based on the terminal bus frequency for a predefined interval subsequent to a large disturbance. A current reference control strategy was proposed in Reference [12] to temporarily provide reactive current support during a terminal voltage dip, and this consequently improved the system transient stability. Reference [13] proposed a synthetic control to provide reactive-current support and to reduce the active-current for transient stability improvement. However, most existing TSCSs are designed for single WT. For a power system integrated with multiple large-scale WTs, how to coordinate these WTs to improve the transient stability has not been investigated.

The nonlinearities inherited in power systems play an important role in TSCS design. To cope with these nonlinearities, many nonlinear control approaches, such as controlled Lyapunov approach $[14,15]$ and feedback linearization approach $[16,17]$, have been implemented. However, those approaches require an accurate model of power systems, which is difficult to obtain in reality. A perturbation estimation (PE) approach provides an alternative to solve this problem [18]. The main idea of PE is to treat the nonlinearities and external disturbances as a perturbation state (PS), and to then try to estimate it by a designed perturbation observer (PO) in real time. With the compensation of the estimated PS, the control design does not require accurate models of power systems, and it consequently provides good robustness to external disturbances.

In this study, a perturbation estimation-based control (PEC) is proposed to control the active-current of WTs for improving power system transient stability. The mutual couplings of generators and WTs are identified using a Kron-reduction approach, and then the control object of each WT is assigned based on the identified mutual couplings. For each WT, an individual controller is developed based on a PE approach to control its active-current for transient stability improvement without requiring accurate models of power systems. Finally, the developed control is tested in the modified IEEE 39-bus system, and the simulation results verify its effectiveness for improving the power system transient stability as well as its robustness to wind speed variation and communication delay in wide area measurement system (WAMS).

The rest of this paper is organized as follows. Section 2 analyzes the mutual couplings between generators and WTs. The design of PEC is presented in Section 3, and Section 4 gives the simulation results of the proposed control approach. Finally, Section 5 concludes the paper.

\section{Mutual Couplings between Generators and WTs}

A power system, consisting of $n$ generators and $m$ WTs, is considered to analyze the effective mutual couplings of generators and WTs as depicted in Figure 1.

For analysis purposes, several assumptions are made as follows:

- System loads are modeled as constant impedance loads and can therefore be absorbed into the node admittance matrix.

- A subtransient model, with subtransient potential in series with subtransient impedance, is considered for synchronous generators.

It should be noted that these assumptions are only for mutual coupling analysis and control strategy design. The designed control strategy is tested in the detailed model of power systems, where the detailed generator model is equipped with an automatic voltage regular (AVR) and governor. The composite load model is also considered. 


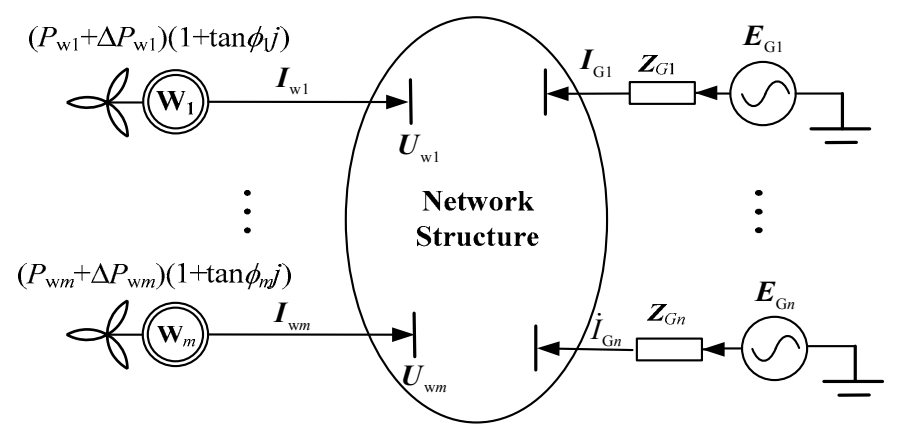

Figure 1. Power system with DFIGs integration.

With the generator subtransient impedances and load impedances included in the node admittance matrix, we obtain

$$
\left[\begin{array}{c}
\boldsymbol{I}_{G} \\
\boldsymbol{I}_{w}
\end{array}\right]=\left[\begin{array}{ll}
\boldsymbol{Y}_{G G} & \boldsymbol{Y}_{G w} \\
\boldsymbol{Y}_{w G} & \boldsymbol{Y}_{w w}
\end{array}\right]\left[\begin{array}{l}
\boldsymbol{E}_{G} \\
\boldsymbol{V}_{w}
\end{array}\right]
$$

where $E_{\mathrm{G}}=\left[E_{\mathrm{G} 1}, \ldots, E_{\mathrm{G} n}\right]^{\mathrm{T}}$ and $I_{\mathrm{G}}=\left[I_{\mathrm{G} 1}, \ldots, I_{\mathrm{G} n}\right]^{\mathrm{T}}$ are the generator potential and current vectors, respectively. $Y_{\mathrm{GG}}, Y_{\mathrm{Gw}}, Y_{\mathrm{wG}}$, and $Y_{\mathrm{ww}}$ are the partitioned matrices of the reduced system admittance matrix with all nodes other than the generator internal nodes and WT terminal nodes eliminated, respectively. $\boldsymbol{V}_{\mathrm{W}}=\left[\boldsymbol{V}_{\mathrm{w} 1}, \ldots, \boldsymbol{V}_{\mathrm{w} m}\right]^{\mathrm{T}}$ is the voltage vector of WT terminal nodes. $\boldsymbol{I}_{\mathrm{W}}=\left[\boldsymbol{I}_{\mathrm{w} 1}, \ldots, \boldsymbol{I}_{\mathrm{w} m}\right]^{\mathrm{T}}$ is the $\mathrm{WT}$ injection current vector.

Kron-reduction is used to determine the effective equivalent admittance of generators and WTs by eliminating the voltage vector $V_{\mathrm{w}}$, and we obtain

$$
\begin{aligned}
& \boldsymbol{I}_{G}=\boldsymbol{Y}_{G G}^{\prime} \boldsymbol{E}_{G}+\boldsymbol{Y}_{G w}^{\prime} \boldsymbol{I}_{w} \\
& \boldsymbol{Y}_{G G}^{\prime}=\boldsymbol{Y}_{G G}+\boldsymbol{Y}_{G w} \boldsymbol{Y}_{w w}^{-1} \boldsymbol{Y}_{w G}, \boldsymbol{Y}_{G w}^{\prime}=\boldsymbol{Y}_{G w} \boldsymbol{Y}_{w w}^{-1}
\end{aligned}
$$

The $i$ th row, $j$ th column element of $Y_{G G}^{\prime}, Y_{G G}^{\prime}(i, j)$, is the Kron-reduced equivalent admittance between generator $i$ and generator $j$, determining the effective mutual coupling of them. The $i$ th row, $j$ th column elements of $\boldsymbol{Y}_{G w}^{\prime}, Y_{G w}^{\prime}(i, j)$, is the Kron-reduced equivalent admittance between generator $i$ and $\mathrm{WT} j$, determining the effective mutual coupling of them.

From (2), the generator current $\boldsymbol{I}_{\mathrm{G}}$ and, further, the generator electrical power $\boldsymbol{P}_{\mathrm{G}}=\operatorname{Re}\left(\boldsymbol{E}_{\mathrm{G}}, \boldsymbol{I}_{\mathrm{G}}\right)$ can be impacted by controlling WT injection $\boldsymbol{I}_{\mathrm{W}}$. Certainly, these impacts are different due to the different mutual couplings between generators and WTs, which can be quantified by the associated Kron-reduced equivalent admittance. The larger the equivalent admittance is, the stronger the mutual coupling is.

\section{Control Design}

\subsection{Control Object Assignation}

Following a severe fault, instability occurs when one or some generators separate from the rest irrecoverably due to the imbalance of their mechanical and electrical power. Mitigating the generator imbalance power by controlling WT injection currents will bring about a transient stability improvement. However, the effects of controlling WT injection currents on mitigating the generator imbalance power are different due to the different effective mutual couplings of generators and WTs, which can be identified by $\boldsymbol{Y}_{G w}^{\prime}$. The stronger the effective mutual couplings are, the more prominent the effects are.

Based on the analysis in Section 2, a control object assignation approach is proposed from the control effect point of view. System generators are clustered into non-lapped generator groups based 
on the mutual couplings of generators and WTs, and each cluster is controlled by a WT. The control object assignation approach consists of 6 steps, as follows:

1. Establish the node admittance matrix of power systems $\boldsymbol{Y}_{\text {sys }}$.

2. Employ the Kron-reduction approach on $\boldsymbol{Y}_{\text {sys }}$ to obtain $\boldsymbol{Y}_{\mathrm{Gw}}$.

3. Transform $\boldsymbol{Y}_{\mathrm{Gw}}{ }_{\mathrm{G}}$ to $\boldsymbol{Y}_{m n}$ by setting the elements of $\boldsymbol{Y}_{\mathrm{Gw}}{ }_{\mathrm{G}}$, whose amplitudes are less than a specified value $\kappa$, as zeros to neglect the weak mutual couplings between generators and WTs.

4. Denote the $i$ th row of $\boldsymbol{Y}_{m n}$ as $\boldsymbol{Y}_{i}$, representing the effective mutual couplings of generator $i$ and each WT.

5. Generator $i$ is clustered into generator group $j$ (Denoted as $G G_{j}$ ), where $j$ is calculated by

$$
\underset{j}{\arg \min }\left\{\operatorname{abs}\left[\boldsymbol{Y}_{m n}(i, j)\right], j=1, \ldots, m\right\}
$$

6. Assign $G G_{j}$ as the control object of WT $j$.

It should be noted that some generators may not be clustered into any group due to their weak mutual couplings with each WT. These generators are not assigned as the control object of any WT, because they can hardly be controlled by any WT.

\subsection{Controlled System Model}

The models of WT and generator used for control design are described in detail in this subsection.

The detailed model of DFIG is given in Figure 2, which consists of a wind turbine, a transmission system, a wound rotor induction generator, and a back-to-back converter. In addition, a crowbar system is used to protect the converter from over-current during the fault, and consequently, this achieves the continuous operation of the DFIG during the transient.

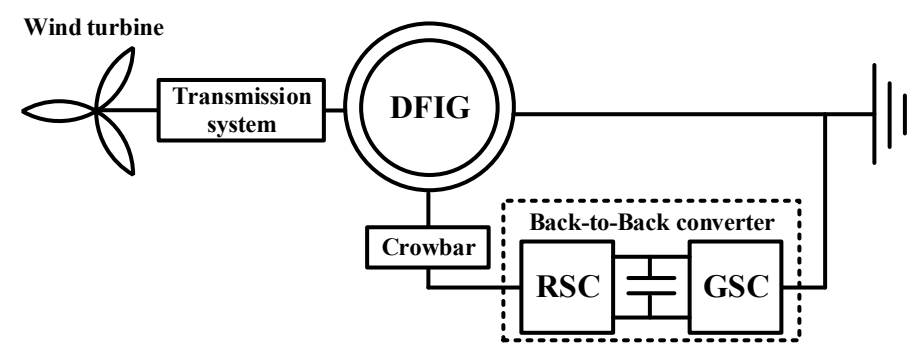

Figure 2. Detailed model of DFIG.

The mechanical power $P_{t}$ captured by a wind turbine from the wind can be expressed as

$$
\left\{\begin{array}{l}
P_{t}=0.5 \rho A v_{\text {wind }}^{3} C_{p}(\lambda, \beta) \\
C_{p}(\lambda, \beta)=0.645\left\{0.00912 \lambda+\left[-5-0.4(2.5+\beta)+116 \lambda_{i}\right] / e^{21 \lambda_{i}}\right\} \\
\lambda_{i}=1 /[\lambda+0.08(2.5+\beta)]-0.035 /\left[1+(2.5+\beta)^{3}\right] \\
\lambda=r \omega_{t} / v_{\text {wind }}
\end{array}\right.
$$

where $\rho, r$, and A are the air density, rotor radio, and rotor-swept area of a wind turbine, respectively; $v_{\text {wind }}, C_{p}, \lambda, \omega_{t}$, and $\beta$ are the wind speed, power coefficient, tip-speed ratio, turbine speed, and pitch angle, respectively. A pitch controller is employed to control the pitch angle $\beta$ for preventing a turbine from over-speed, as shown in Figure 3. 


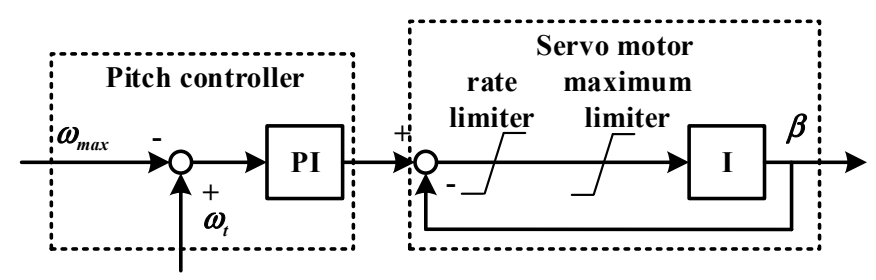

Figure 3. Pitch controller scheme.

A transmission system, consisting of a low-speed drive shaft, gearbox, and a high-speed drive shaft, transmits the mechanical power $P_{m}$ to the wound induction generator, which can be modeled as a two-mass shaft model in (5).

$$
\left\{\begin{array}{l}
2 H_{t} \frac{d \omega_{t}}{d t}=P_{t}-K_{s} \theta_{s}-D_{s}\left(\omega_{t}-\omega_{g}\right)-D_{t} \omega_{t} \\
2 H_{g} \frac{d \omega_{g}}{d t}=K_{s} \theta_{s}+D_{s}\left(\omega_{t}-\omega_{g}\right)-D_{g} \omega_{g}-P_{g} \\
\frac{d \theta_{s}}{d t}=\omega_{s}\left(\omega_{t}-\omega_{g}\right)
\end{array}\right.
$$

where $H_{t}, D_{t}$ and $P_{t}$ are the inertia time constant, damping constant, and mechanical torque of a wind turbine, respectively; $H_{g}, D_{g}, \omega_{g}$, and $P_{g}$ are the inertia time constant, damping constant, speed, and mechanical torque of a DFIG, respectively; $K_{s}, D_{s}, \theta_{s}$, and $\omega_{s}$ are the shaft stiffness, damping constant, torsional twist, and synchronous speed, respectively.

The wound induction generator model in the $d-q$ reference frame can be expressed as follows:

$$
\left\{\begin{array}{l}
V_{s d}=R_{s} i_{s d}-\omega_{s} \psi_{s q}+d \psi_{s d} / d t \\
V_{s q}=R_{s} i_{s q}+\omega_{s} \psi_{s q}+d \psi_{s q} / d t \\
V_{r d}=R_{r} i_{r d}-\omega_{s l i p} \psi_{r q}+d \psi_{r d} / d t \\
V_{r q}=R_{r} i_{r q}+\omega_{s l i p} \psi_{r d}+d \psi_{r q} / d t \\
\psi_{s d}=L_{s} i_{s d}+L_{m} i_{r d} \\
\psi_{s q}=L_{s} i_{s q}+L_{m} i_{r q} \\
\psi_{r d}=L_{m} i_{s d}+L_{r} i_{r d} \\
\psi_{r q}=L_{m} i_{s q}+L_{r} i_{r q}
\end{array}\right.
$$

where $\omega_{\text {slip }}=\omega_{s}-\omega_{g}$ is the slip of DFIG. $V_{s d}, V_{s q}$ and $V_{r d}, V_{r q}$ represent the $d$-axis and q-axis components of the stator voltage and rotor voltage, respectively. $i_{s d}, i_{s q}$ and $i_{r d}, i_{r q}$ represent the $d$-axis and q-axis components of the stator current and rotor current, respectively. $\psi_{s d}, \psi_{s q}$ and $\psi_{r d}, \psi_{r q}$ represent the $d$-axis and q-axis components of the stator flux and rotor flux, respectively. $L_{s}, L_{r}$, and $L_{m}$ represent the stator inductor, rotor inductor, and the mutual inductor of stator and rotor, respectively. $R_{s}$ and $R_{r}$ represent the stator resistance and rotor resistance, respectively.

A back-to-back converter consists of a gird side converter (GSC) and a rotor side converter (RSC), where the GSC maintains the DC voltage of the capacitor and regulates the reactive power of the GSC exchanged with the grid, and the RSC performs the maximum power point tracking (MPPT) control and regulates the stator reactive power injected into the grid. The standard vector control technique is applied on both the RSC and GSC, and the $d$-axis of the synchronously rotating frame is oriented along the stator voltage $V_{\mathrm{W}}$ as shown in Formula (7), where the stator resistance $R_{\mathrm{S}}$ is neglected for being much smaller than its reactance $L_{s}$.

$$
\left\{\begin{array} { l } 
{ V _ { s d } = - \omega _ { s } \psi _ { s q } = - \omega _ { s } ( L _ { s } i _ { s q } + L _ { m } i _ { r q } ) = V _ { w } } \\
{ V _ { s q } = \omega _ { s } \psi _ { s d } = \omega _ { s } ( L _ { s } i _ { s d } + L _ { m } i _ { r d } ) = 0 }
\end{array} \Rightarrow \left\{\begin{array}{l}
i_{s d}=L_{m} i_{r d} / L_{s} \\
i_{s q}=-\left(V_{w}+L_{s} L_{m} i_{r q}\right) / L_{s} \omega_{s}
\end{array}\right.\right.
$$

Therefore, the injection current of DFIG $j$ can be expressed

$$
\boldsymbol{I}_{w, j}=I_{s d, j}+I_{s q, j} i
$$


where the $d$-axis stator current $i_{s d, j}$ and $q$-axis stator current $i_{s q, j}$ can be controlled by the rotor active-current $i_{r d, j}$ and rotor reactive-current $i_{r q, j}$ respectively as shown in (9).

$$
\left\{\begin{array}{l}
i_{s d, j}=L_{m, j} i_{r d, j} / L_{s, j} \\
i_{s q, j}=-\left(V_{w w j}+L_{s, j} L_{m, j} i_{r q, j}\right) / L_{s, j} \omega_{s}
\end{array}\right.
$$

where $L_{s, j}$ and $L_{m, j}$ represent the stator inductor and mutual induction of stator and rotor of DFIG $j$, represectively. $V_{\mathrm{w} j}$ is the amplitude of the terminal voltage $V_{w j}$.

A typical converter controller is shown in Figure 4, which consists of a fast time-scale current controller, a medium time-scale DC voltage controller, and a slow time-scale speed controller. The proposed PEC is integrated in the RSC, and the auxiliary signal $u_{j}$, derived from the PEC, is added on the active-current reference $I_{r d r e f, j}$ to modulate the active-current of DFIG for improving power system transient stability.

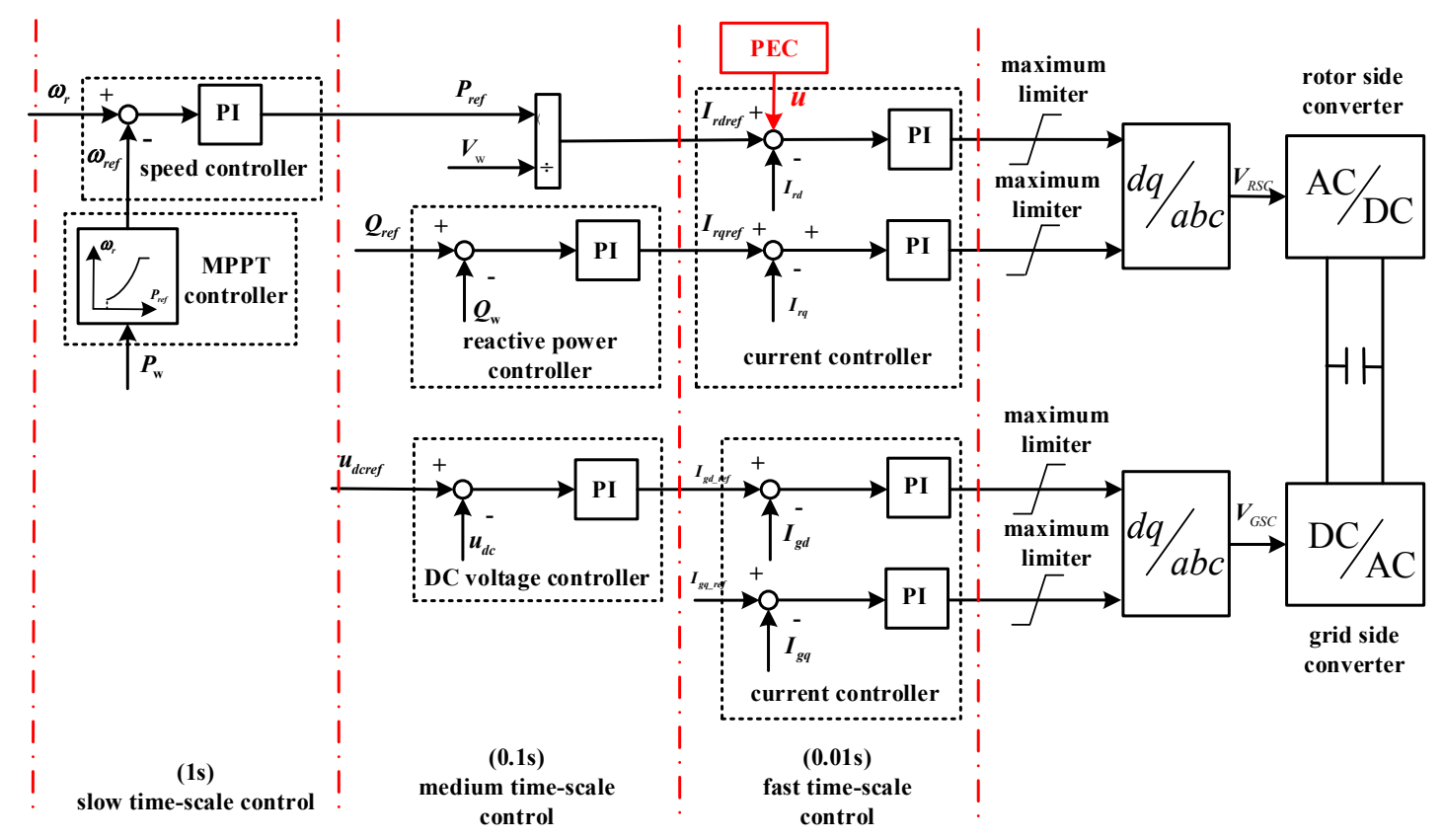

Figure 4. Scheme of converter controller.

In terms of transient stability, the time-scale of interest (approximate $0.1-1 \mathrm{~s}$ ) is much slower than the time-scale of the current controller (approximate 0.01-0.1 s), as shown in Figure 4 and demonstrated in [19]. Therefore, the dynamics of the current controller can be omitted, and the $d$-axis current $I_{r d}$ can be considered as instantaneously tracking their reference values, as follows:

$$
u_{j}+I_{r d r e f, j}=I_{r d, j}
$$

The swing Formula models, which link the rotor acceleration to the imbalance between the mechanical power supplied by the governor and the electrical power, are considered to describe the dynamics of generators in power systems, as follows:

$$
\left\{\begin{array}{l}
\frac{d \delta_{i}}{d t}=\omega_{i} \\
\frac{d \omega_{i}}{d t}=\frac{1}{M_{i}}\left(P_{m i}-P_{G i}\right), \quad i=1, \ldots, n
\end{array}\right.
$$

where $M_{i}$ is the inertial constant. $\delta_{i}$ and $\omega_{i}$ are the rotor angle and rotor speed of generator $i$, respectively. $P_{\mathrm{m} i}$ and $P_{\mathrm{G} i}$ are the mechanical and electrical power of generator $i$, respectively. 
$P_{\mathrm{G} i}$ can be expressed as

$$
P_{G i}=\operatorname{Real}\left[\boldsymbol{E}_{G i}\left(\boldsymbol{I}_{G i}\right)^{*}\right]
$$

Substituting Formula (8) and (9) into (12), we obtain

$$
\begin{aligned}
P_{G i} & =\operatorname{Re}\left\{\boldsymbol{E}_{G i} \sum_{k=1}^{n}\left[\boldsymbol{Y}_{G G}^{\prime}(i, k) \boldsymbol{E}_{G k}\right]^{*}+\boldsymbol{E}_{G i} \sum_{j=1}^{m}\left[\boldsymbol{Y}_{G w}^{\prime}(i, j) \boldsymbol{I}_{w j}\right]^{*}\right\}=P_{G i G}+P_{G i w} \\
P_{G i G} & =\operatorname{Re}\left[\boldsymbol{E}_{G i} \sum_{k=1}^{n}\left(\boldsymbol{Y}_{G G}^{\prime}(i, k) \boldsymbol{E}_{G k}\right)^{*}\right] \\
P_{G i w} & =\operatorname{Re}\left[\boldsymbol{E}_{G i} \sum_{j=1}^{m}\left(\boldsymbol{Y}_{G w}^{\prime}(i, j) \boldsymbol{I}_{w j}\right)^{*}\right] \\
& =\sum_{j=1}^{m}\left(c_{P i j} I_{s d, j}-c_{Q i j} I_{s q, j}\right)=\sum_{j=1}^{m}\left\{c_{P i j}\left(-\frac{L_{m, j}}{L_{s, j}}\left(i_{d r r e f, j}+u_{j}\right)\right)-c_{Q i j} I_{s q, j}\right\} \\
c_{P i j} & =E_{G i} Y_{G w}^{\prime}(i, j) \cos \left(\delta_{i}-\alpha_{i j}\right), c_{Q i j}=E_{G i} Y_{G w}^{\prime}(i, j) \sin \left(\delta_{i}-\alpha_{i j}\right)
\end{aligned}
$$

where $E_{\mathrm{G} i}$ and $\delta_{\mathrm{G} i}$ are the amplitude and phase of $E_{\mathrm{G} i}$, respectively. $V_{\mathrm{w} j}$ and $\theta_{\mathrm{w} j}$ are the amplitude and phase of $V_{\mathrm{w} j}$, respectively. $Y_{\mathrm{Gw}}(i, j)$ and $\alpha_{i j}$ are the amplitude and phase of $Y_{\mathrm{Gw}}(i, j)$, respectively.

From Formula (13), $P_{G i}$ consists of two terms: $P_{\mathrm{GiG}}$ and $P_{\mathrm{Giw}}$, which represent the exchanged power with the other generators and WTs, respectively. It is clear that the active-power of generator $i$ can be regulated by controlling either the injection active-current or reactive-current of WTs. Considering the fact that the transient stability is high relative to the active-power, only the active-current of WTs is considered in PEC design.

From (11)-(13), the model consisting of $n_{j}$ generators within $\mathrm{GG}_{j}$ and $\mathrm{WT} j$ can be expressed as

$$
\left\{\begin{aligned}
\frac{d \delta_{1}}{d t} & =\omega_{1} \\
\frac{d \omega_{1}}{d t} & =\frac{1}{M_{1}}\left\{P_{m 1}-P_{G 1 G}-\sum_{j=1}^{m}\left[-\frac{c_{P 1 j} L_{m, 1}}{L_{s, 1}}\left(I_{d r r e f, 1}+u_{1}\right)-c_{Q 1 j} I_{s q, 1}\right]\right\}_{j} \\
\vdots & \\
\frac{d \delta_{n_{j}}}{d t} & =\omega_{n_{j}} \\
\frac{d \omega_{n_{j}}}{d t} & =\frac{1}{M_{n_{j}}}\left\{P_{m n_{j}}-P_{G n_{j} G}-\sum_{j=1}^{m}\left[-\frac{c_{P_{n_{j}} L_{m, n_{j}}}}{L_{s, n_{j}}}\left(I_{d r r e f, n_{j}}+u_{n_{j}}\right)-c_{Q n_{j}} I_{s q, n_{j}}\right]\right\} \\
y_{j} & =h_{j}\left(\delta_{1}, \omega_{1}, \ldots, \delta_{n_{j}}, \omega_{n_{j}}\right)
\end{aligned}\right.
$$

where $n_{j}$ is the total number of generators in $G G_{j} . y_{j}=h_{j}\left(\delta_{1}, \omega_{1}, \ldots, \delta_{n j}, \omega_{n j}\right)$ is the output variable as illustrated in Formula (15).

\subsection{Design of PEC}

The transient stability control object is to restore the generator rotor speeds back to the synchronous speed $\omega_{s}$, consequently making generators synchronize again. Therefore, in this paper, the output variable $y_{j}$ is selected as the average value of rotor speeds of generators within $G G_{j}$, as follows:

$$
y_{j}=\frac{1}{n_{j}} \sum_{i \in G G_{j}} \omega_{i}
$$

To employ a PE approach, the linearization model of a power system should be established by calculating the derivative of the variable $y_{j}$ until the control input signal $u_{\mathrm{w} j}$ appears

$$
\frac{d y_{j}}{d t}=\frac{1}{n_{j}} \sum_{i=1}^{n_{j}}\left\{\frac{1}{M_{i}}\left[P_{m i}-P_{G i G}-\sum_{j=1}^{m}\left(-\frac{c_{P i j} L_{m, j}}{L_{s, j}}\left(I_{d r r e f}+u_{j}\right)-c_{Q i j} I_{q}\right)\right]\right\}
$$


For Formula (16), a PS $\psi_{j}$ is defined in Formula (17) to represent the comprehensive perturbation of the nonlinearities and parameter variation.

$$
\psi_{j}=\frac{1}{n_{j}} \sum_{i=1}^{n_{j}}\left\{\frac{1}{M_{i}}\left[P_{m i}-P_{G i G}-\sum_{j=1}^{m}\left(-\frac{c_{P i j} L_{m, j}}{L_{s, j}}\left(I_{d r r e f}+u_{j}\right)-c_{Q i j} I_{q}\right)\right]\right\}-b_{0 j} u_{j}
$$

where $b_{0 j}$ is a positive constant.

Substituting Formula (16) into (15), we obtain the following:

$$
\left\{\begin{array}{l}
d y_{j} / d t=\psi_{j}+b_{0 j} u_{j} \\
d \psi_{j} / d t=\lambda
\end{array}\right.
$$

where $\lambda$ is the derivative of $\psi_{j}$.

Instead of calculating $\psi_{j}$ according to its definition in Formula (18), a PO is designed for Formula (17) to estimate $\psi_{j}$ in real time.

$$
\left\{\begin{array}{l}
d \widetilde{y}_{j} / d t=\widetilde{\psi}_{j}+b_{0 j} u_{j}-l_{j 1}\left(\widetilde{y}_{j}-y_{j}\right) \\
d \widetilde{\psi}_{j} / d t=-l_{j 2}\left(\widetilde{y}_{j}-y_{j}\right)
\end{array}\right.
$$

where $\left[\widetilde{y}_{j}, \widetilde{\psi}_{j}\right]$ are the estimations of $\left[z_{j 1}, \psi_{j}\right]$, respectively. $l_{j i}(i=1,2)$ are the observer coefficients.

From Formula (18) and (19), the error dynamics of a PO can be obtained by

$$
\left\{\begin{array}{l}
d \widetilde{y}_{j} / d t=\widetilde{\psi}_{j}+b_{0 j} u_{j}-l_{j 1}\left(\widetilde{y}_{j}-y_{j}\right) \\
d \widetilde{\psi}_{j} / d t=-l_{j 2}\left(\widetilde{y}_{j}-y_{j}\right)
\end{array}\right.
$$

The observer coefficients should be set properly so as to place the pole of Equation (20) at the desired location in the open left-half complex plane. By choosing a positive constant as the pole of Equation (21), the observer coefficients can be easily obtained.

$$
s^{2}+l_{j 1} s+l_{j 2}=\left(s+\lambda_{j}\right)^{2}
$$

where $\lambda_{j}$ is the chosen pole.

To achieve quick and accurate estimation of PS, the pole $\lambda_{j}$ of (21) should be set large enough. However, an overlarge $\lambda_{j}$ will increase the sensitivity to the noise in signaling. Therefore, the selection of $\lambda_{j}$ must make a trade-off between the tracking performance and the noise tolerance.

With the estimated PS, the final control law is given in (22), where the estimated PS $\widetilde{\psi}_{j}$ is used to reject the comprehensive disturbance.

$$
u_{w j}=\left(-k_{j} \widetilde{z}_{j 1}-\widetilde{\psi}_{j}\right) / b_{0 j}
$$

where $k_{j}$ is a positive constant.

The larger the value of $k_{j}$ is, the better performance PEC provides. However, an overlarge $k_{j}$ will exploit more kinetic energy stored in the rotating mass, which aggravates the fluctuation of rotor speed and may violate the specified speed range of $[0.7,1.25]$ p.u. Therefore, the selection of $k_{j}$ should make a trade-off between the control performance and the rotor speed fluctuation.

To prevent a PEC from activating in cases of small rotor speed deviation due to the small disturbance occurred in the power system, a dead-band block is designed as follows:

$$
y_{j}= \begin{cases}0 & \left|y_{j}\right|<\varepsilon_{j} \\ y_{j} & \left|y_{j}\right| \geq \varepsilon_{j}\end{cases}
$$

where $\varepsilon_{j}$ is a small positive constant, which is set by the power system operator. 
Moreover, before adding the output of PEC on the active-current reference, the control output of a PEC should pass through a limiter to prevent overmodulation. A PEC exploits the available kinetic energy of a WT to improve system transient stability. However, the available kinetic energy depends on the turbine speed, which varies with the wind speed under a MPPT control mode. The overmodulation of a PEC may cause the turbine speed to violate the permissible range, consequently causing WT tripping. In this paper, the output control of PEC $u_{j}$ is limited within the range of $[-0.3,0.3]$, based on the simulation results. How to select appropriate configurations of limits under different wind speed conditions requires further research, which is not the focus of this paper.

To clearly illustrate the structure of the proposed PEC, a block diagram is given in Figure 5.

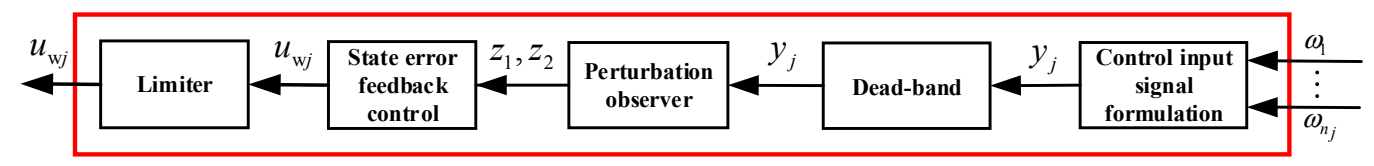

Figure 5. The block diagram of PEC.

\section{Simulation Study}

\subsection{Test System}

To test the performance of the developed PEC, a modified IEEE 39-bus system integrated with four DFIG-based wind farms (WFs) of rated capacity $600 \mathrm{MW}$ was established on a DIgSILENT platform (version 2018 SP1, DIgSILENT/PowerFactory Company, Berlin, Germany), as depicted in Figure 6. The four WFs operated at different wind speed conditions, as shown in Table 1, and the total wind power accounted for $18.9 \%$ of the overall power generation. In practice, each WF contained a large number of small capacity WTs. Establishing the detailed model of every WF would result in a heavy simulation burden and increase the simulation time. Therefore, the aggregation technique, which is commonly used in the published papers [11], was taken by modeling a WF as a single WT of equivalent capacities. The configurations of the WT were taken from Formula [20].

Table 1. Wind speeds and active-power of different WFs.

\begin{tabular}{ccccc}
\hline Wind Farm & $\mathbf{W}_{\mathbf{1}}$ & $\mathbf{W}_{\mathbf{2}}$ & $\mathbf{W}_{\mathbf{3}}$ & $\mathbf{W}_{\mathbf{4}}$ \\
\hline Wind speed & $8.62 \mathrm{~m} / \mathrm{s}$ & $7.54 \mathrm{~m} / \mathrm{s}$ & $8.12 \mathrm{~m} / \mathrm{s}$ & $9.08 \mathrm{~m} / \mathrm{s}$ \\
Active-power & $300 \mathrm{MW}$ & $200 \mathrm{MW}$ & $250 \mathrm{MW}$ & $350 \mathrm{MW}$ \\
\hline
\end{tabular}

The subtransient models were considered for generators. All generators other than $G_{1}$, which is an equivalent external generator, were equipped with AVR type IEEE-ET1 and speed governor type IEEE-G1. The composite load models consisting of 50\% constant load and 50\% induction motor load were considered. 


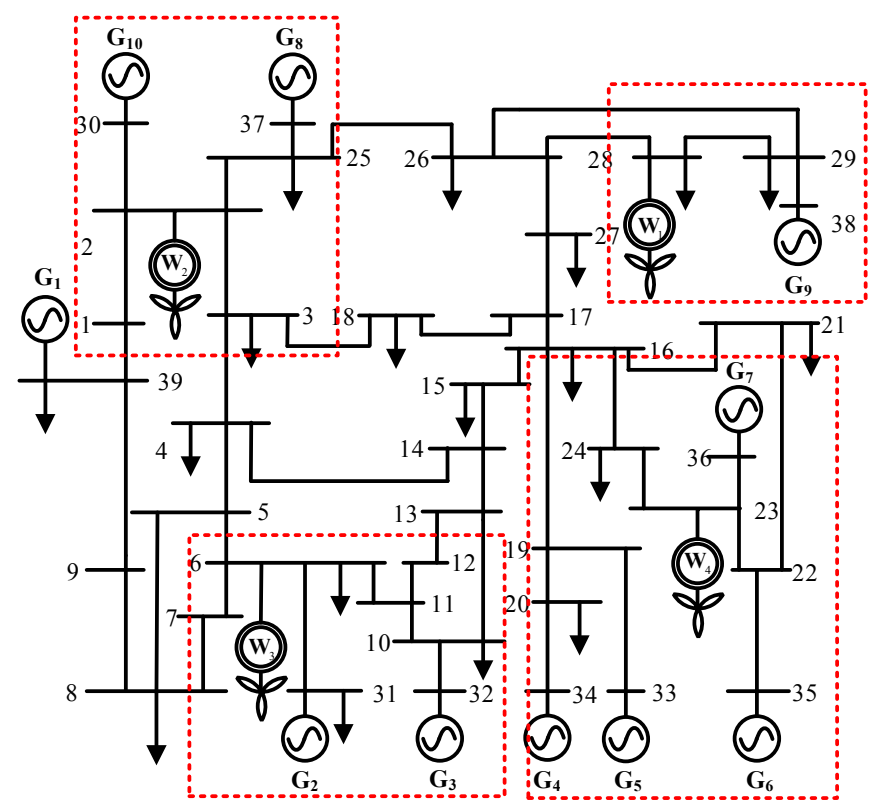

Figure 6. The modified IEEE 39-bus system.

\subsection{Configuration of PEC}

The element amplitudes of $\boldsymbol{Y}_{G w}^{\prime}$ were calculated as shown in Formula (24) to analyze the mutual couplings of generators and WTs. It was clear, for each WT, that there only existed a small number of strong coupled generators. By setting $\mathrm{K}=10^{-2}$, generators were clustered into four generator groups $\left\{\mathrm{GG}_{1}\right\},\left\{\mathrm{GG}_{2}\right\},\left\{\mathrm{GG}_{3}\right\}$, and $\left\{\mathrm{GG}_{4}\right\}$ consisting of $\left\{\mathrm{G}_{9}\right\},\left\{\mathrm{G}_{8}, \mathrm{G}_{10}\right\},\left\{\mathrm{G}_{2}, \mathrm{G}_{3}\right\}$, and $\left\{\mathrm{G}_{4}, \mathrm{G}_{5}, \mathrm{G}_{6}, \mathrm{G}_{7}\right\}$, respectively, and these groups were assigned as the control object of each WT.

After control object assignation, an individual controller was developed for each WT as described in Section 3.1. There were only 3 parameters that required configuration for the PEC: the observer pole $\lambda_{j}$ and the positive constants $b_{0 j}$ and $k_{j}$. These parameters were selected by trial and error, and all controllers took the same configuration: $\lambda_{j}=10, b_{0 j}=1, k_{j}=10$. Therefore, the observer coefficients $l_{j 1}$ and $l_{j 2}$ were set as 20 and 100 , respectively.

$$
\operatorname{abs}\left(Y_{G w}^{\prime}\right)=\left[\begin{array}{cccc}
0.08 & 0.12 & 0.14 & 0.03 \\
0.01 & 0.16 & 2.27 & 0.08 \\
0.01 & 0.23 & 3.57 & 0.11 \\
0.03 & 0.08 & 0.01 & 1.89 \\
0.06 & 0.01 & 0.07 & 2.08 \\
0.04 & 0.03 & 0.15 & 3.51 \\
0.03 & 0.02 & 0.09 & 3.26 \\
0.15 & 3.42 & 0.14 & 0.06 \\
4.63 & 0.72 & 0.03 & 0.08 \\
0.34 & 2.67 & 0.83 & 0.02
\end{array}\right] \times 10^{-2}
$$

\subsection{General Performance of PEC}

The performance of the PEC was evaluated by applying a three-phase-to-ground fault at the middle of the transmission line between buses 15 and 16 at $1 \mathrm{~s}$ and then isolating the fault line at $1.12 \mathrm{~s}$. The rotor angles and rotor speeds of all generators without and with the PEC were compared, as shown in Figure 7, which clearly illustrates the effectiveness of the proposed PEC in improving the transient stability of power systems. 

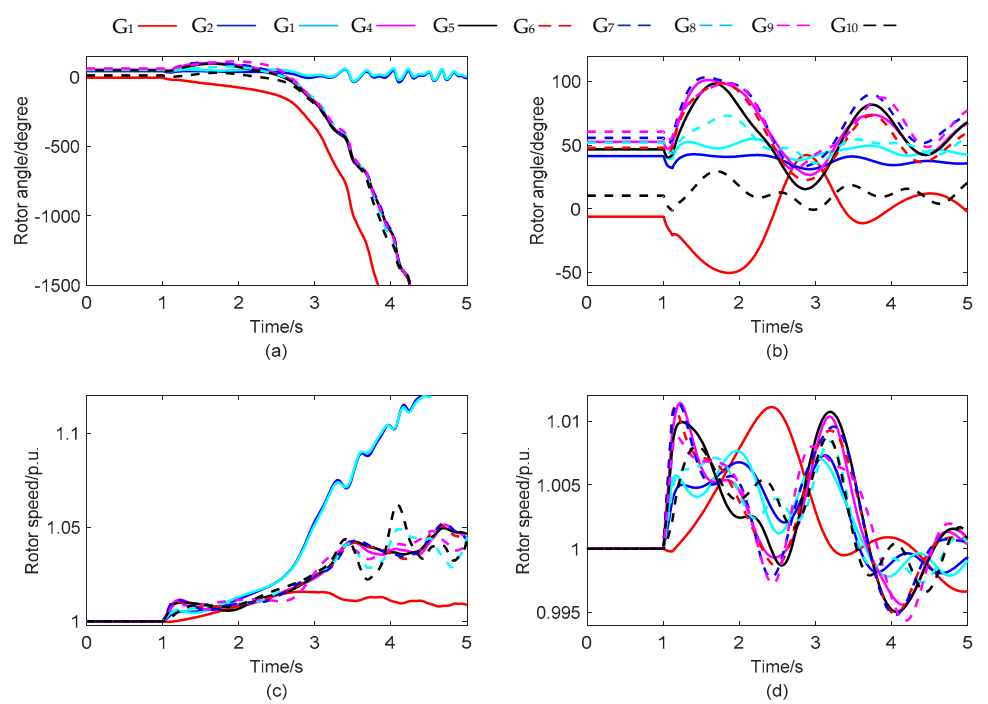

Figure 7. Dynamics of the generator (a) rotor angle and (c) rotor speed without PEC; Dynamics of the generator $(\mathbf{b})$ rotor angle and $(\mathbf{d})$ rotor speed with PEC.

The dynamics of all WTs without and with the PEC are compared in Figure 8. The active-powers of WTs were regulated by controlling the active-current of WTs with the PEC to improve the system transient stability, as shown in Figure 8a,b. It should be noted that the crowbar of DFIG $\mathrm{W}_{4}$ was activated at $1.01 \mathrm{~s}$ to protect converter from over-current, and then it was removed at $1.12 \mathrm{~s}$ after the fault clearance, consequently achieving the continuous operation of the WT. The developed PEC was activated at $1.13 \mathrm{~s}$ after the removal of the crowbar to control the active-current of the WT. The PEC exploited the kinetic energy stored in the rotating mass by changing the turbine speed. However, due to the large inertia of the rotating mass, a small speed fluctuation occured, which did not threaten the stable operation of the WT (shown in Figure 8d). The dynamics of terminal voltage are compared in Figure 8e,f, which illustrates that the developed PEC does not violate the specified voltage limits ([0.7-1.2] p.u.) during or following a severe fault.

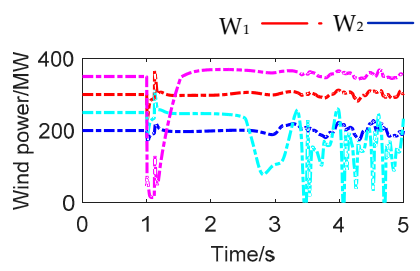

(a)

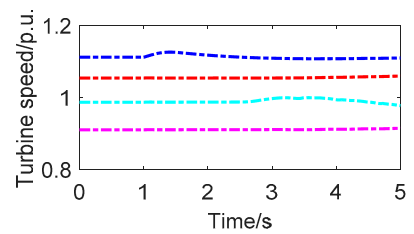

(c)

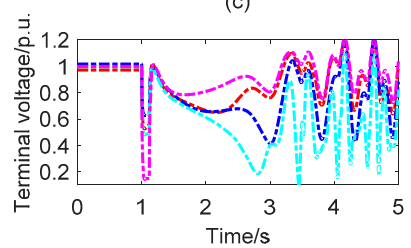

(e)

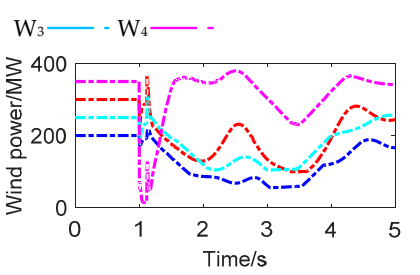

(b)

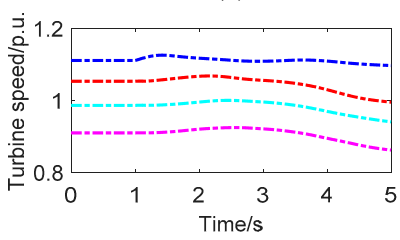

(d)

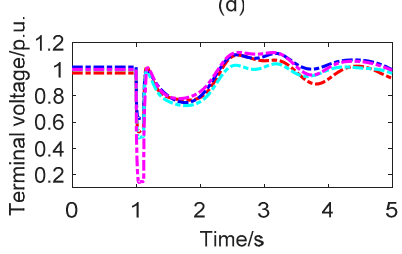

(f)

Figure 8. DFIG dynamics of (a) wind power, (c) turbine speed, (e) terminal voltage without PEC; DFIG dynamics of $(\mathbf{b})$ wind power, $(\mathbf{d})$ turbine speed, $(\mathbf{f})$ terminal voltage with PEC. 
The critical clearing time (CCT) is a widely used metric in evaluating the system transient stability. The CCTs with and without the PEC under different fault conditions are compared in Table 2. It can be seen that the transient stability margin with the PEC increases effectively compared to that without the PEC, which verifies the effectiveness of the proposed PEC.

Table 2. Comparison of CCTs.

\begin{tabular}{cccccc}
\hline Wind Condition & Fault & No Control & PEC & $\begin{array}{c}\text { PEC with 100 } \\
\text { ms Delay }\end{array}$ & $\begin{array}{c}\text { PEC with 200 } \\
\text { ms Delay }\end{array}$ \\
\hline \multirow{3}{*}{ Constant } & Line 15-16 & $112 \mathrm{~ms}$ & $136 \mathrm{~ms}$ & $130 \mathrm{~ms}$ & $128 \mathrm{~ms}$ \\
& Line 2-3 & $130 \mathrm{~ms}$ & $172 \mathrm{~ms}$ & $168 \mathrm{~ms}$ & $166 \mathrm{~ms}$ \\
& Line 5-6 & $186 \mathrm{~ms}$ & $230 \mathrm{~ms}$ & $226 \mathrm{~ms}$ & $226 \mathrm{~ms}$ \\
\hline \multirow{3}{*}{ Variable (shown in Figure 9) } & Line 15-16 & $106 \mathrm{~ms}$ & $132 \mathrm{~ms}$ & $128 \mathrm{~ms}$ & $126 \mathrm{~ms}$ \\
& Line 2-3 & $123 \mathrm{~ms}$ & $168 \mathrm{~ms}$ & $164 \mathrm{~ms}$ & $164 \mathrm{~ms}$ \\
& Line 5-6 & $160 \mathrm{~ms}$ & $220 \mathrm{~ms}$ & $218 \mathrm{~ms}$ & $214 \mathrm{~ms}$ \\
\hline
\end{tabular}

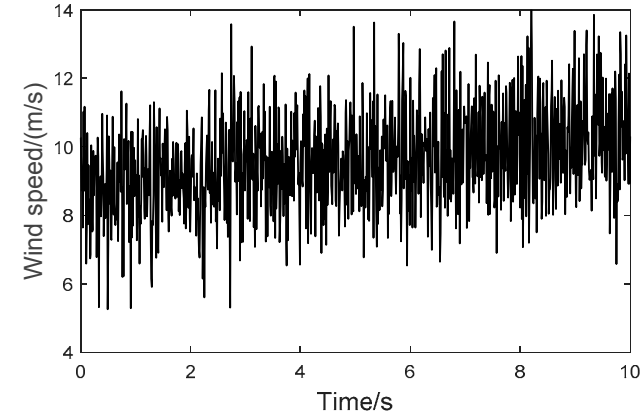

(a)

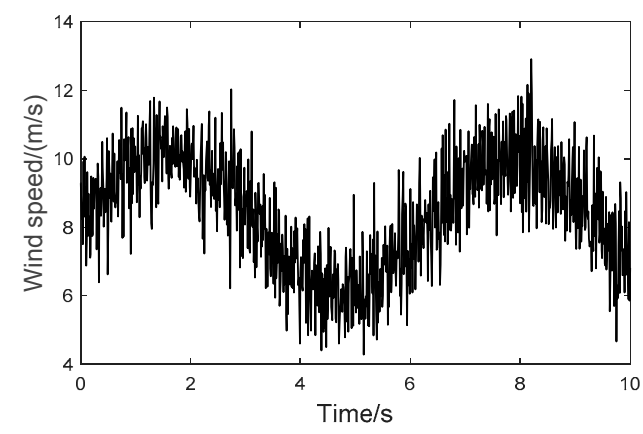

(c)

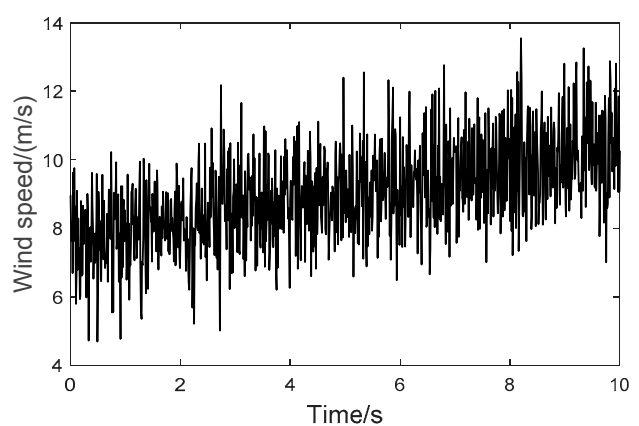

(b)

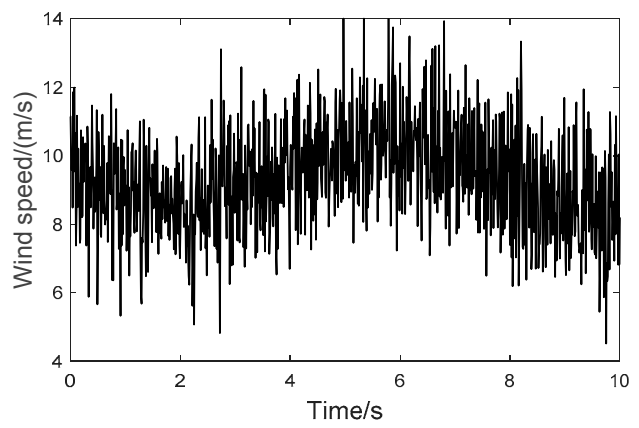

(d)

Figure 9. Wind speed of (a) $\mathrm{W}_{1} ;$ (b) $\mathrm{W}_{2} ;$ (c) $\mathrm{W}_{3}$; and (d) $\mathrm{W}_{4}$.

\subsection{Robustness to Wind Speed Fluctuation}

In the above simulation contingency, wind speed was assumed as constant during the transient, while wind speed varies at all time in nature. This section describes the investigation results for a case in which wind speeds of $W_{1}, W_{2}$, and $W_{3}$ varied, as shown in Figure 9. The fault, as described in Section 4.3, was applied again. Figure 10 compares the dynamics of the generator rotor angle and the rotor speed with and without the PEC, which verifies the effectiveness of the proposed PEC on improving the system transient stability under variable wind speed conditions. The dynamics of the WT under variable wind conditions are given in Figure 11. The PEC exploited the kinetic energy of the WT to improve the transient stability, consequently causing rotor speed fluctuation. However, the rotor speed fluctuation was still within the admissible range of $[0.7,1.25]$ p.u., which did not threaten its stable operation. As shown in Figure 11f, the terminal voltage dynamics with the PEC were within the admissible range. The CCTs under variable wind speed conditions are shown in Table 2, where a 
larger stable margin was obtained with PEC. Therefore, the good robustness of the proposed PEC to wind speed fluctuation has been verified.

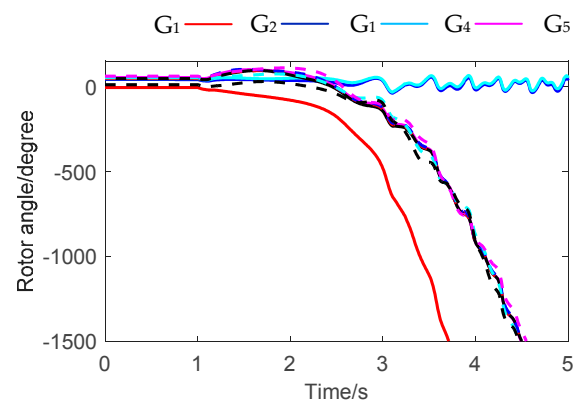

(a)

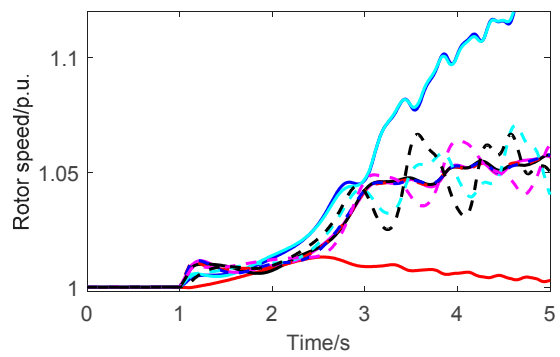

(c)

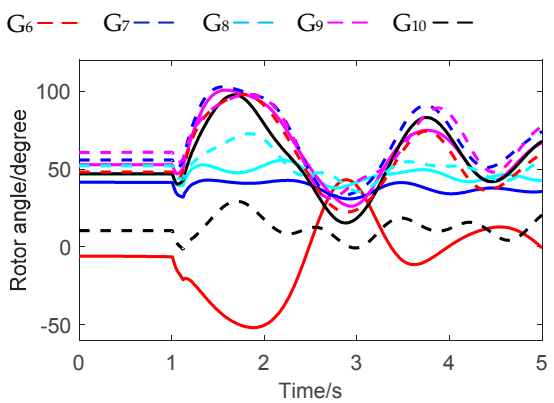

(b)

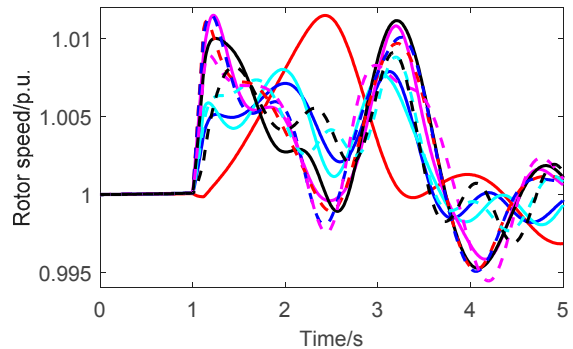

(d)

Figure 10. Dynamics of the generator (a) rotor angle and (b) rotor speed without PEC; Dynamics of the generator $(\mathbf{c})$ rotor angle and $(\mathbf{d})$ rotor speed with PEC.

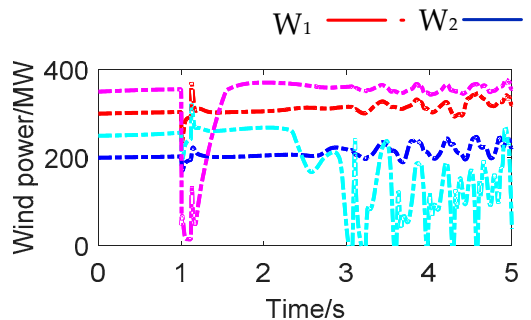

(a)

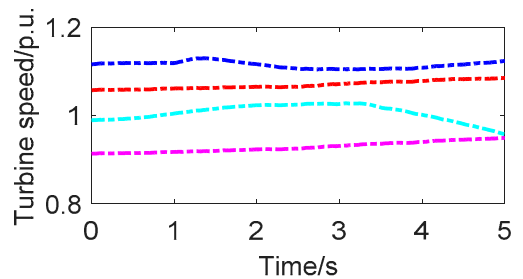

(c)

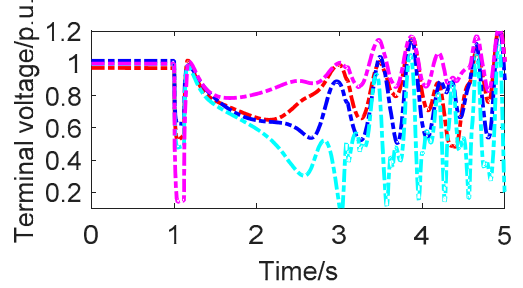

(e)

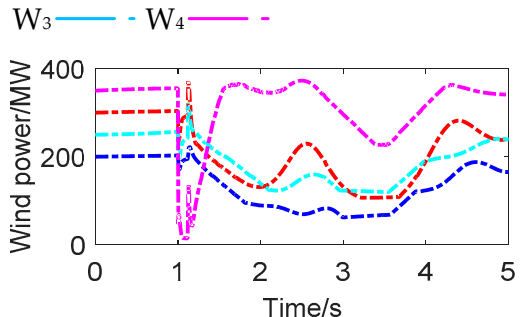

(b)

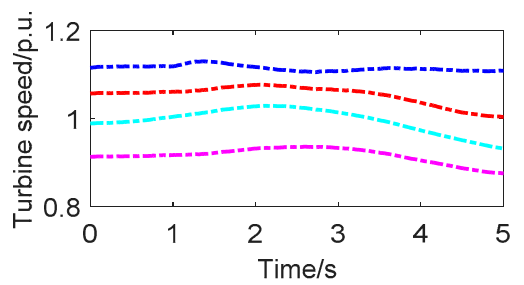

(d)

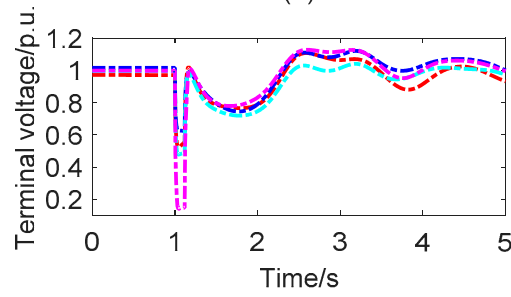

(f)

Figure 11. WT dynamics of (a) wind power; (c) turbine speed; and (e) terminal voltage without PEC; DFIG dynamics of (b) wind power; (d) turbine speed; and (f) terminal voltage with PEC. 


\subsection{Robustness to the Communication Delay}

Since the developed PEC takes wide-area measurements as its control input signal, the communication delay in the WAMS had an adverse effect on the control performance of the PEC. In reality, the communication delay in the WAMS using optical fiber was usually within the range of 80-150 ms [21]. The dynamics of generator rotor angle and rotor speed with $100 \mathrm{~ms}$ and $200 \mathrm{~ms}$ communication delays are shown in Figure 12, respectively, which are similar to the dynamics with no communication delay, as shown in Figure 7.
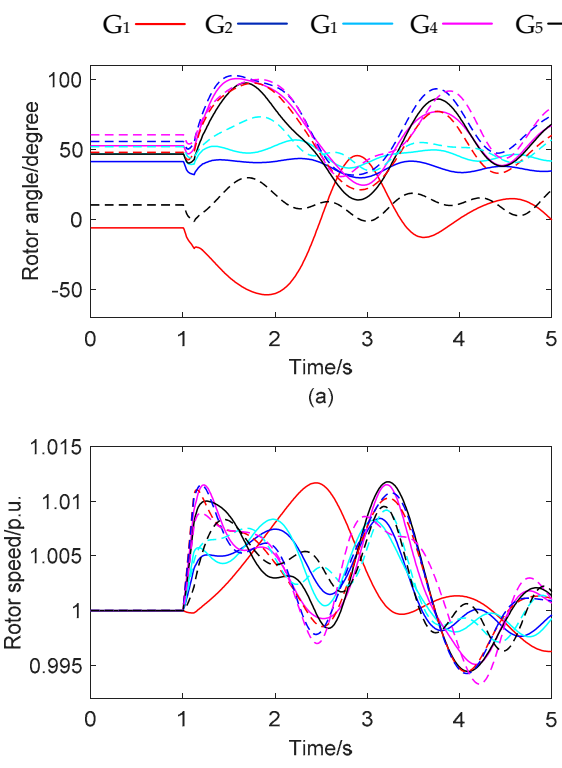

(c)

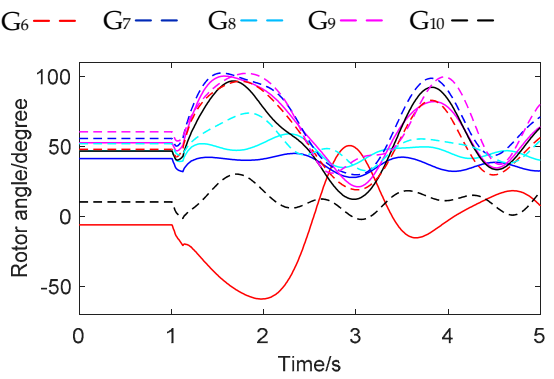

(b)

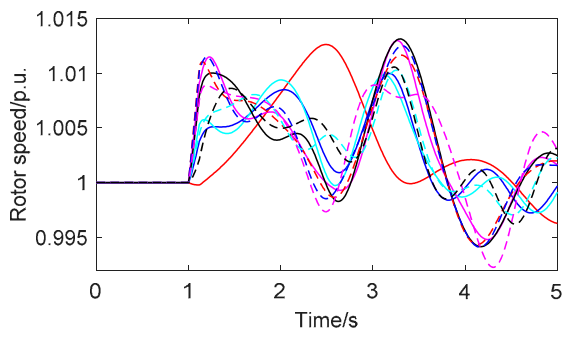

(d)

Figure 12. Dynamics of generator (a) rotor angle; (c) rotor speed with $100 \mathrm{~ms}$ delay; dynamics of generator $(\mathbf{b})$ rotor angle; $(\mathbf{d})$ rotor speed with $200 \mathrm{~ms}$ delay.

The CCTs with different communication delays are given in Table 2. The increase of communication delay has little impact on the performance of the PEC. Therefore, the proposed PEC is robust to the possible and extreme delays in the communication network.

\section{Conclusions}

This paper proposes an active-current control for large-scale WTs to improve power system transient stability. The control object of each WT is assigned based on the identified effective mutual couplings of generators and WTs using a Kron-reduction approach, and an individual controller is designed for each WT using a PE technique to control its active-current for improving the system transient stability. The design of the PEC does not require an accurate model of power systems. The simulation results, carried in the modified IEEE 39-bus system, verify the effectiveness of the proposed PEC on the system transient stability improvement under different fault conditions as well as its robustness to wind speed variation and communication delay in WAMS.

Author Contributions: P.S. and L.G. propose the main idea of the paper; P.S. implement the mathematical; Z.H., L.W. and Z.J. implement the simulation analysis and analyzed the data. This paper is written by P.S. and is revised by L.G.

Funding: This work is supported by China Southern Power Grid (grant no. CSGTRC-K163007).

Conflicts of Interest: The authors declare no conflict of interest. 


\section{References}

1. Kundur, P. Power System Stability and Control; McGraw-Hill: New York, NY, USA, 1994.

2. Wang, H.; Zhang, B.H.; Hao, Z.G. Response based emergency control system for power system transient stability. Energies 2015, 8, 13508-13520. [CrossRef]

3. Xu, X.; Zhang, H.Z.; Li, C.G.; Liu, Y.T.; Li, W.; Terzija, V. Optimization of the event-driven emergency load-shedding considering transient security and stability constraints. IEEE Trans. Power Syst. 2017, 32, 2581-2592. [CrossRef]

4. Zhang, Z.; Yang, H.; Yin, X.; Han, J.; Wang, Y.; Chen, G. A load-shedding model based on sensitivity analysis in on-line power system operation risk assessment. Energies 2017, 11, 727. [CrossRef]

5. Zhou, Y.Z.; Huang, H.Y.; Xu, Z.; Hua, W.; Yang, F.Y.; Liu, S. Wide-area measurement system-based transient excitation boosting control to improve power system transient stability. IET Gener. Transm. Distrib. 2015, 9, 845-854. [CrossRef]

6. Vittal, E.; O'Malley, M.; Keane, A. Rotor angle stability with high penetration of wind generation. IEEE Trans. Power Syst. 2012, 27, 353-362. [CrossRef]

7. Edrah, M.; Lo, K.L.; Anaya-Lara, O. Impacts of high penetration of DFIG wind turbines on rotor angle stability of power systems. IEEE Trans. Sustain. Energy 2015, 6, 759-766. [CrossRef]

8. Cardenas, R.; Pena, R.; Alepuz, S.; Asher, G. Overview of control systems for the operation of DFIGs in wind energy applications. IEEE Trans. Ind. Electron. 2013, 60, 2776-2798. [CrossRef]

9. Liu, Z.Y.; Liu, C.R.; Li, G.Y.; Liu, Y.; Liu, Y.L. Impact study of PMSG-based wind power generation on power system transient stability using EEAC theory. Energies 2015, 8, 13419-13441. [CrossRef]

10. Hossain, M.J.; Pota, H.R.; Mahmud, M.A.; Ramos, R.A. Investigation of the impacts of large-scale wind power penetration on the angle and voltage stability of power system. IEEE Syst. J. 2012, 6, 76-84. [CrossRef]

11. Mitra, A.; Chatterjee, D. Active power control of DFIG-based wind farm for improvement of transient stability of power systems. IEEE Trans. Power Syst. 2016, 31, 82-93. [CrossRef]

12. Werise, B. Impact of K-factor and active current reduction during fault-ride-through of generating units connected via voltage-sourced converters on power system stability. IET Renew. Power Gener. 2015, 9, 25-36.

13. Ullah, N.R.; Thiringer, T.; Karlsson, D. Voltage and transient stability support by wind farms complying with the E.ON Netz grid code. IEEE Trans. Power Syst. 2007, 22, 1647-1656. [CrossRef]

14. Eriksson, R. On the centralized nonlinear control of HVDC systems using Lyapunov Theory. IEEE Trans. Power Deliv. 2013, 28, 1156-1163. [CrossRef]

15. Eriksson, R. Coordinated control of multiterminal DC grid power injections for improved rotor-angle stability based on Lyapunov theory. IEEE Trans. Power Deliv. 2014, 29, 1789-1797. [CrossRef]

16. Mahmud, M.A.; Pota, H.R.; Aldeen, M.; Hossain, MJ. Partial feedback linearizing excitation controller for multimachine power systems to improve transient stability. IEEE Trans. Power Syst. 2014, 29, 561-571. [CrossRef]

17. Lu, Q.; Sun, Y.Z. Nonlinear stabilizing control of multimachine systems. IEEE Trans. Power Syst. 1989, 4, 236-241. [CrossRef]

18. Han, J.Q. From PID to active disturbance rejection control. IEEE Trans. Ind. Electron. 2009, 56, 900-906. [CrossRef]

19. Hu, J.B.; Huang, Y.H.; Wang, D.; Yuan, H.; Yuan, X.M. Modeling of grid connected DFIG-based wind turbines for DC-link voltage stability analysis. IEEE Trans. Sustain. Energy 2015, 6, 1325-1336. [CrossRef]

20. Ma, Y.; Liu, J.; Liu, H.; Zhao, S. Active-reactive additional damping control of a doubly-fed induction generator based on active disturbance rejection control. Energies 2018, 11, 1314. [CrossRef]

21. Naduvathuparambil, B.; Valenti, M.C.; Feliachi, A. Communication delay in wide-area measurement systems. In Proceedings of the Thirty-Fourth Southeastern Symposium on System Theory (Cat. No.02EX540), Huntsville, AL, USA, 19 March 2002; pp. 118-122.

(C) 2018 by the authors. Licensee MDPI, Basel, Switzerland. This article is an open access article distributed under the terms and conditions of the Creative Commons Attribution (CC BY) license (http:/ / creativecommons.org/licenses/by/4.0/). 